\title{
A Class of Selective CIC FIR Filter Functions
}

\author{
Vlastimir D. Pavlović, Dejan N. Milić, Member, IEEE, and Biljana P. Stošić, Member, IEEE
}

\begin{abstract}
A new class of modified selective multiplerless Cascaded-Integrator-Comb (CIC) finite impulse response (FIR) filter functions with application in modern communication systems, which have superior performances compared to the classical CIC filter functions are presented here. A few design examples that illustrate the properties of the proposed filter class are also presented. Novel filter functions have a minimum attenuation of $166.52 \mathrm{~dB}, \mathbf{2 5 8 . 5 6}$ $\mathrm{dB}$ and $350.46 \mathrm{~dB}$, respectively. The achieved improvements versus classical CIC filters are $29.84 \mathrm{~dB}, 34.90 \mathrm{~dB}$ and 39.82 $\mathrm{dB}$, respectively.
\end{abstract}

Keywords - CIC filters, FIR filters, linear phase, multiplierless structure, selective filters.

\section{INTRODUCTION}

$\mathrm{T}$ HE term "Cascaded-Integrator-Comb (CIC)" filters was first reported in the early $1980 \mathrm{~s}$ by E.B. Hogenauer [1]. Software defined radio has found an important place in modern communication systems where the majority of signal processing is performed in the digital domain. In software radio systems, sample rate changes can be very large. Such a requirement leads to large order digital filters, which can easily become a bottleneck in the overall system design. A CIC finite impulse response (FIR) filter that uses only additions/subtractions can be used to reduce the computational demands. Many papers have addressed the problem of some modifications of the classical CIC filters for sample rate conversion in software defined radio systems [2]-[4]. A number of demonstrated characteristics make the structures proposed in [2]-[3] a good candidate for software defined radio applications. Modified CIC filters are ideal for applications in which high sampling rates make the use of multipliers a computationally expensive option. This technique is especially useful for FPGA design [4] where multipliers are avoided because of the large silicon area required. The use of CIC filters is also important in applications in which large rate change factors require large amounts of coefficient storage or fast

Paper received August 25, 2014; revised October 1, 2014; accepted October 2, 2014. Date of publication July 15, 2015. The associate editor coordinating the review of this manuscript and approving it for publication was Prof. Branimir Reljin.

This work has been partially supported by the Ministry for Education, Science and Technological Development of Serbia, project numbers TR32023, III44006 and TR32052.

Vlastimir D. Pavlović and Dejan N. Milić are with the University of Niš, Faculty of Electronic Engineering, Serbia (phone: 381-18-529-206, 381-18-529-304; e-mails: vlastimir.pavlovic@telfak.ni.ac.rs, dejan.milic@elfak.ni.ac.rs).

Corresponding Biljana P. Stošić is with the University of Niš, Faculty of Electronic Engineering, Serbia (phone: 381-18-529-303, e-mail: biljana.stosic@elfak.ni.ac.rs). impulse response generation and the memory is either unavailable or too slow to perform the desired application. Other solutions proposed in the literature give some modified filter structures for sigma-delta analog-to-digital converters [5]-[6].

Because of the disadvantages of a CIC FIR filter (a not flat passband and a high passband drop), some solutions given in the literature try to improve the classical CIC magnitude response characteristic. Some authors use a compensation filter in the cascade with the original filter [7]-[12]. Then, some other use sharpening technique [13] or design a new class of filter functions as shown in [14][18].

The aim of this paper is to present a novel class of CIC FIR filter functions which is based on cascading CIC sections of different lengths (non-identical CIC sections). Design examples of the novel designed class of CIC filter functions are used to validate their characteristics by comparing them with those of the classical CIC filters under fair conditions: the same number of cascaded sections and the same group delay. The results show a substantial performance improvement of the proposed filter class compared to the classical CIC filters.

\section{ClassicAl CIC FILTER}

The conventional CIC FIR filters are well known in literature. The normalized CIC FIR filter function of one section in $z$-domain is defined with

$$
H(N, z)=\frac{1}{N} \cdot\left(1+z^{-1}+z^{-2}+\cdots+z^{-(N-1)}\right)=\frac{1-z^{-N}}{N \cdot\left(1-z^{-1}\right)}
$$

There are two basic building blocks: a comb and an integrator. The filter function of a comb in $z$-domain is $H_{c}(N, z)=\left(1-z^{-N}\right)$, therefore a difference equation in time domain is $y_{c}(n)=x(n)-x(n-N)$. Function $H_{i}(z)=1 /\left(1-z^{-1}\right)$ represents the filter function of an integrator in $z$-domain and $y_{i}(n)=x(n)+y(n-1)$ is its time domain representation.

A poor magnitude characteristic of the CIC filter composed of one section, Eq. (1), is improved by cascading several identical CIC filters. The classical CIC FIR filter function of normalized amplitude response characteristic, represented in the $z$-domain, is defined as

$$
H(N, K, z)=\left(\frac{1-z^{-N}}{N \cdot\left(1-z^{-1}\right)}\right)^{K}
$$

where $N$ is the decimation factor, and $K$ is the number of sections (identical cascaded CIC filters of one section) [1]. 
The frequency response characteristic of CIC FIR filter function can be written in the form

$$
H\left(N, K, z=e^{j \omega}\right)=e^{-j K(N-1) \omega / 2} \cdot\left(\frac{\sin (N \omega / 2)}{N \cdot \sin (\omega / 2)}\right)^{K}
$$

\section{NOVEl Class OF CIC FIR FILTER FUNCTIONS}

The novel class is designed as a cascade of four nonidentical CIC FIR filter functions: one function $H(N-1, z)$, two functions $H(N, z)$, and one function $H(N+1, z)$, of the form given by (1), as well as seven cascaded non-identical CIC FIR filter sections which are repeated an integer number of times.

The filter function of normalized amplitude response characteristic of a designed novel class of CIC FIR filter functions can be written in a non-recursive form as follows $H(N, K, L, z)=\left(\frac{1}{N-1} \sum_{r=0}^{N-2} z^{-r}\right)\left(\frac{1}{N} \sum_{r=0}^{N-1} z^{-r}\right)\left(\frac{1}{N} \sum_{r=0}^{N-1} z^{-r}\right)$.

$\left(\frac{1}{N+1} \sum_{r=0}^{N} z^{-r}\right) \cdot\left[\left(\frac{1}{N-3} \sum_{r=0}^{N-4} z^{-r}\right)\left(\frac{1}{N-2} \sum_{r=0}^{N-3} z^{-r}\right)\left(\frac{1}{N-1} \sum_{r=0}^{N-2} z^{-r}\right)\right]^{L}$. $\left[\left(\frac{1}{N} \sum_{r=0}^{N-1} z^{-r}\right)\left(\frac{1}{N+1} \sum_{r=0}^{N} z^{-r}\right)\left(\frac{1}{N+2} \sum_{r=0}^{N+1} z^{-r}\right)\left(\frac{1}{N+3} \sum_{r=0}^{N+2} z^{-r}\right)\right]^{L}$

where $N$ and $L$ are free integer parameters, and $K=7 L+4$. This form is suitable for hardware realization, because it is unconditionally stable.

The novel class of CIC FIR filter functions is designed based on the classical CIC filters, by spreading the delays in the CIC filter comb stages. The delays are set around one specific value of $N$. The recursive form of a novel class of CIC FIR filter functions with a normalized amplitude response characteristic is

$H(N, K, L, z)=$

$=\frac{1-z^{-(N-1)}}{(N-1) \cdot\left(1-z^{-1}\right)} \frac{1-z^{-N}}{N \cdot\left(1-z^{-1}\right)} \frac{1-z^{-N}}{N \cdot\left(1-z^{-1}\right)} \frac{1-z^{-(N+1)}}{(N+1) \cdot\left(1-z^{-1}\right)}$.

$\left(\frac{1-z^{-(N-3)}}{(N-3) \cdot\left(1-z^{-1}\right)} \frac{1-z^{-(N-2)}}{(N-2) \cdot\left(1-z^{-1}\right)} \frac{1-z^{-(N-1)}}{(N-1) \cdot\left(1-z^{-1}\right)}\right)^{L}$.

$\left(\frac{1-z^{-N}}{N \cdot\left(1-z^{-1}\right)} \frac{1-z^{-(N+1)}}{(N+1) \cdot\left(1-z^{-1}\right)} \frac{1-z^{-(N+2)}}{(N+2) \cdot\left(1-z^{-1}\right)} \frac{1-z^{-(N+3)}}{(N+3) \cdot\left(1-z^{-1}\right)}\right)^{L}$ and $K=7 L+4$.

\section{A. Frequency Response Characteristic}

The frequency response of the designed novel class of FIR filter functions is obtained by evaluating the filter function in the $z$-plane at the sample points defined by setting $z=e^{j \omega}$, where $\omega=2 \pi \cdot f$ has units of radians per second. Using Euler's identity, it can be separated into a real-valued magnitude $A(N, K, L, \omega)$ and a real-valued phase angle $\varphi(N, K, L, \omega)$ for each frequency $\omega$,

$$
H\left(N, K, L, z=e^{j \omega}\right)=e^{j \cdot \varphi(N, K, L, \omega)} \cdot A(N, K, L, \omega)
$$

where the parameter $K=7 L+4$.

The normalized amplitude response characteristic of the proposed filter functions is defined in the form
$A(N, K, L, \omega)=$

$=\frac{\sin ((N-1) \omega / 2)}{(N-1) \cdot \sin (\omega / 2)} \frac{\sin (N \omega / 2)}{N \cdot \sin (\omega / 2)} \frac{\sin (N \omega / 2)}{N \cdot \sin (\omega / 2)} \frac{\sin ((N+1) \omega / 2)}{(N+1) \cdot \sin (\omega / 2)}$.

$\left(\frac{\sin ((N-3) \omega / 2)}{(N-3) \cdot \sin (\omega / 2)} \frac{\sin ((N-2) \omega / 2)}{(N-2) \cdot \sin (\omega / 2)} \frac{\sin ((N-1) \omega / 2)}{(N-1) \cdot \sin (\omega / 2)}\right)^{L}$.

$\left(\frac{\sin (N \omega / 2)}{N \cdot \sin (\omega / 2)} \frac{\sin ((N+1) \omega / 2)}{(N+1) \cdot \sin (\omega / 2)} \frac{\sin ((N+2) \omega / 2)}{(N+2) \cdot \sin (\omega / 2)} \frac{\sin ((N+3) \omega / 2)}{(N+3) \cdot \sin (\omega / 2)}\right)^{L}$

and $K=7 L+4$.

The normalized magnitude response characteristic $\left|H\left(N, K, L, e^{j \omega}\right)\right|$ is obtained as the absolute value of the normalized amplitude response characteristic $A(N, K, L, \omega)$ given by (7).

The linear phase response characteristic of the proposed novel class of the modified CIC FIR filter has the form $\varphi(N, K, L, \omega)=-(N-1) \cdot K \cdot \omega / 2+2 \cdot v \cdot \pi, v=0,1,2, \ldots$ and $K=7 L+4$.

The constant group delay response characteristic of the proposed novel class of the modified CIC FIR filter functions is expressed as

$\tau(N, K, L, \omega)=-d \varphi(N, K, L, \omega) / d \omega=(N-1) \cdot K / 2$,

and $K=7 L+4$.

It is independent of the frequency. For $N$ being an odd number, the group delay is an integer multiple of $K / 2$. If $N$ is an even number, the group delay is equal to an integer plus half a multiple of $K / 2$.

\section{B. Selection of the Design Parameters}

The choice of free integer parameters $N$ and $L$ is done in the same way as for CIC filters, there are the same restrictions on the group delay response. The parameter $K$ can take different integer values, $K=7 L+4$.

The attenuation in the stopband region is closely related to the parameter $L$. By increasing $L$ for a constant value of $N$, a higher stopband attenuation is achieved.

The constant group delay $\tau$ is equal for the classical CIC filters (Eq. (3)) and the novel modified CIC filter functions (Eq. (9)). The values of constant group delay for different values of parameters $N, L$ and $K=7 L+4$ are given in Table 1 .

TABLE 1. GROUP DELAy $\tau(\omega)$ FOR $N \in\{6,7, \ldots, 11\}$, $L \in\{1,2,3\}$ AND $K=7 L+4$.

\begin{tabular}{|c|l|l|l|l|l|l|l|}
\hline \multicolumn{2}{|c|}{$N$} & 6 & 7 & 8 & 9 & 10 & 11 \\
\hline$\tau[s]$ & $\begin{array}{l}L=1, \\
K=11\end{array}$ & 27.5 & 33 & 38.5 & 44 & 49.5 & 55 \\
\cline { 2 - 8 } & $\begin{array}{l}L=2, \\
K=18\end{array}$ & 45 & 54 & 63 & 72 & 81 & 90 \\
\cline { 2 - 8 } & $\begin{array}{l}L=3, \\
K=25\end{array}$ & 62.5 & 75 & 87.5 & 100 & 112.5 & 125 \\
\hline
\end{tabular}

\section{FREQUENCY RESPONSE CHARACTERISTICS}

A few illustrative examples are designed and simulations are conducted in order to compare the frequency response characteristics of the classical CIC filter functions and the novel designed classes of selective CIC FIR filter functions. These functions are compared in fair conditions: the same level of constant group delay $\tau$, as well as the number of delay elements. The novel filter 
functions $H(N, K, L, z)$ from Eq. (5) and the classical CIC filters $H(N, K, z)$ from Eq. (2) have the same number of cascaded sections $K$, with a difference that the CIC filters have an identical structure in all cascades, and the proposed functions have a cascade connections of nonidentical CIC sections. The choice of free parameters $N$ and $L$ is done in the same way as for CIC filters, there are the same restrictions on the group delay response. The parameter $K$ can take values $K=7 L+4$. Generally, the main task of the comparison was to vary the free parameters and to compare obtained filter characteristics.

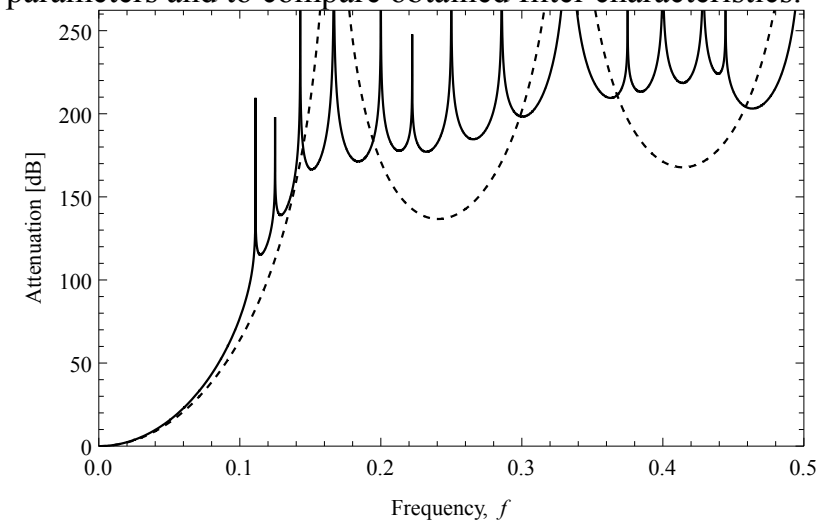

(a) Classical CIC filter for $K=11$ (dashed line), novel class of CIC filter for $K=11, L=1$ (solid line)

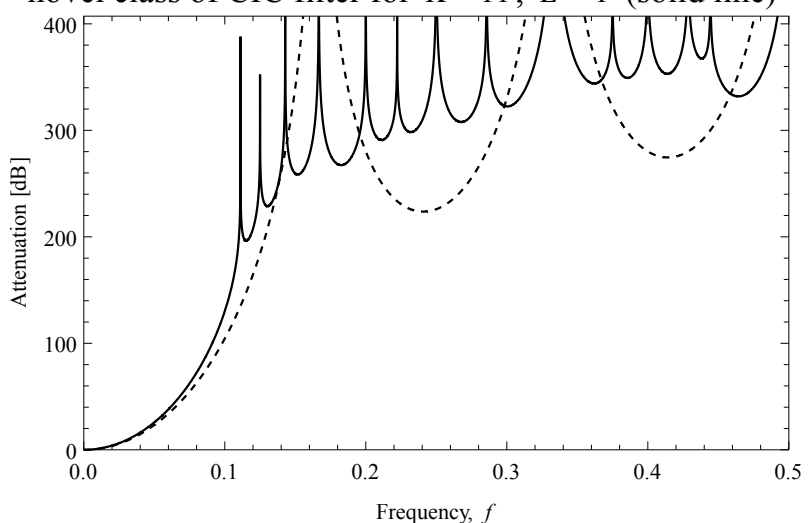

(b) Classical CIC filter for $K=18$ (dashed line), novel class of CIC filter for $K=18, L=2$ (solid line)

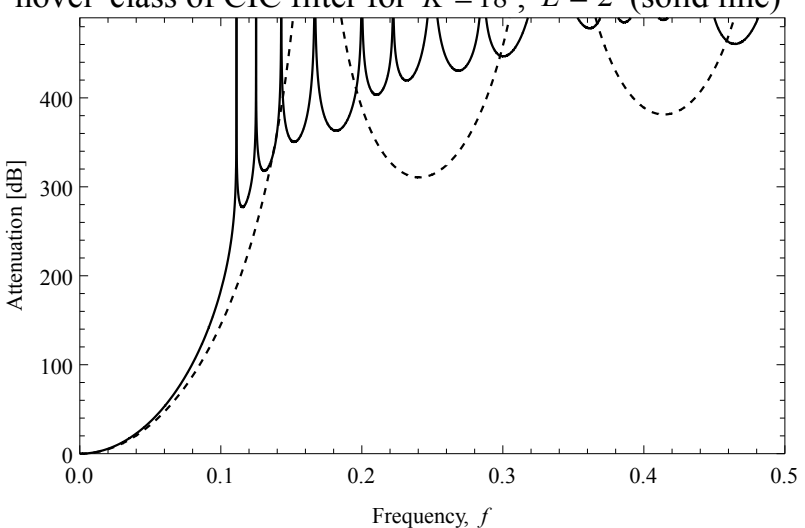

(c) Classical CIC filter for $K=25$ (dashed line), novel class of CIC filter for $K=25, L=3$ (solid line).

Fig. 1. Comparison of normalized magnitude response characteristics in $\mathrm{dB}$ of classical CIC filter (dashed lines), and proposed novel class of CIC FIR filter functions (solid lines), for $N=6$.

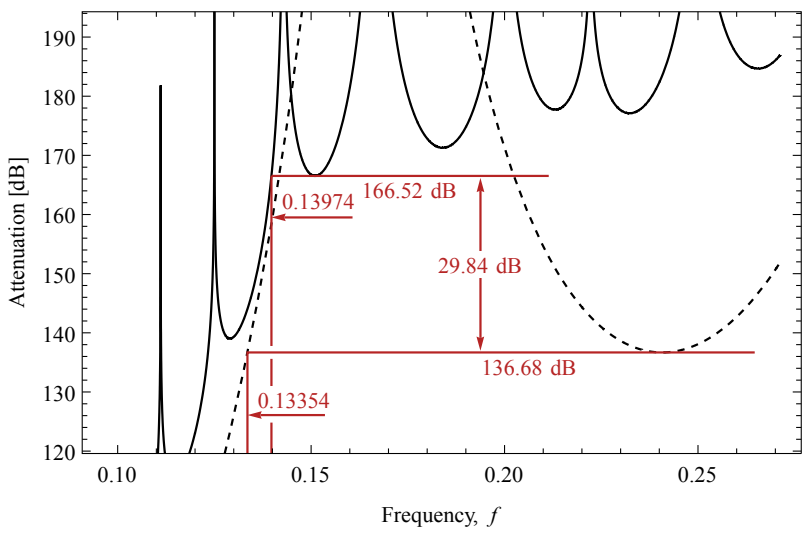

(a) Classical CIC filter for $K=11$ (dashed line), novel class of CIC filter for $K=11, L=1$ (solid line)

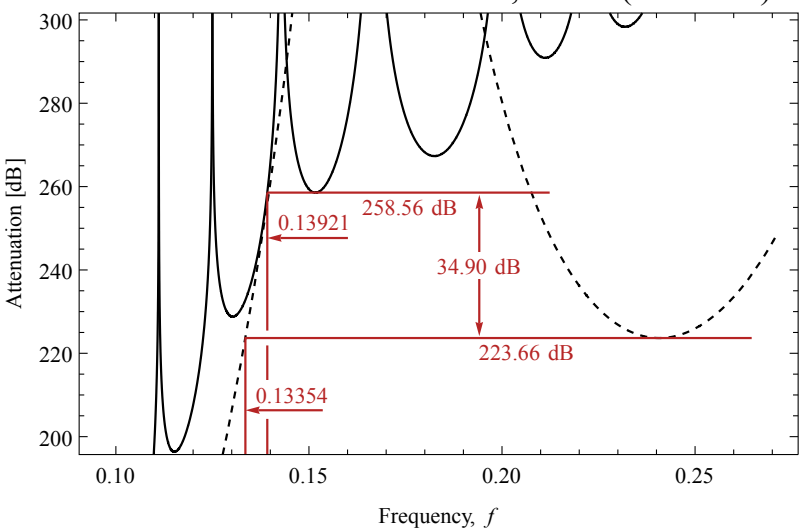

(b) Classical CIC filter for $K=18$ (dashed line), novel class of CIC filter for $K=18, L=2$ (solid line)

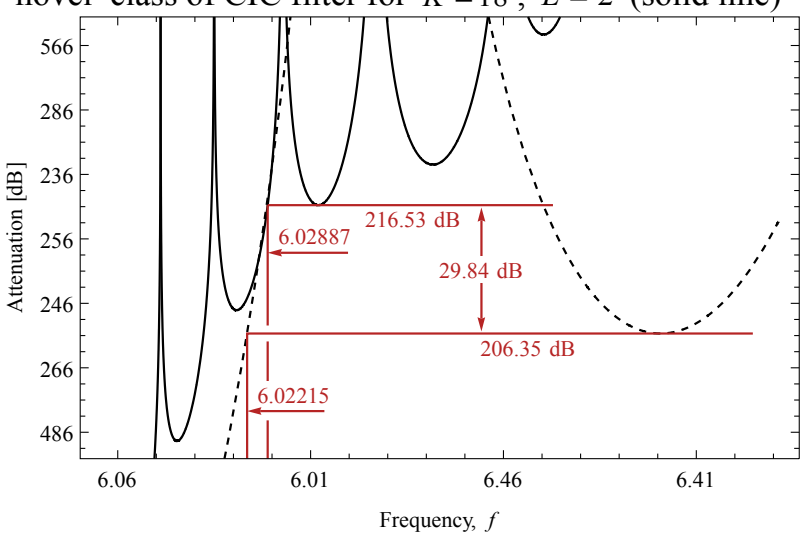

(c) Classical CIC filter for $K=25$ (dashed line), novel class of CIC filter for $K=25, L=3$ (solid line)

Fig. 2. Zooms of normalized magnitude response characteristics in $\mathrm{dB}$ of classical CIC filter (dashed lines), and proposed novel class of CIC FIR filter functions (solid lines), for $N=6$.

A detailed analysis of the normalized magnitude response characteristics in $\mathrm{dB}$, defined for the classical CIC filters as $\alpha_{C I C}(f)=-20 \cdot \log \left|H\left(N, K, e^{j 2 \pi f}\right)\right|$ and for the novel class of CIC FIR filter functions as $\alpha(f)=-20 \cdot \log \left|H\left(N, K, L, e^{j 2 \pi f}\right)\right|$, versus normalized frequency $f=\omega /(2 \pi)$, is depicted in Fig. 1. The maximum attenuation in the passband is $\alpha_{\max }=0.28 \mathrm{~dB}$. 


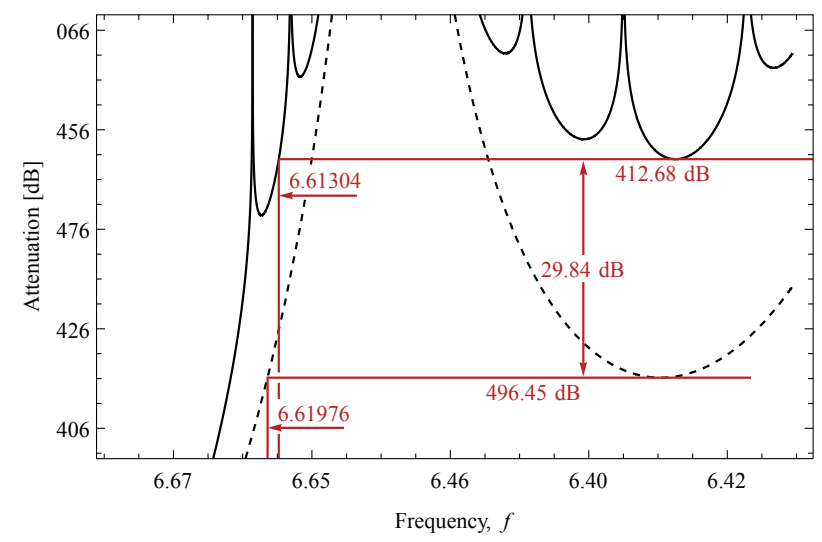

(a) Classical CIC filter for $K=11$ (dashed line), novel class of CIC filter for $K=11, L=1$ (solid line)

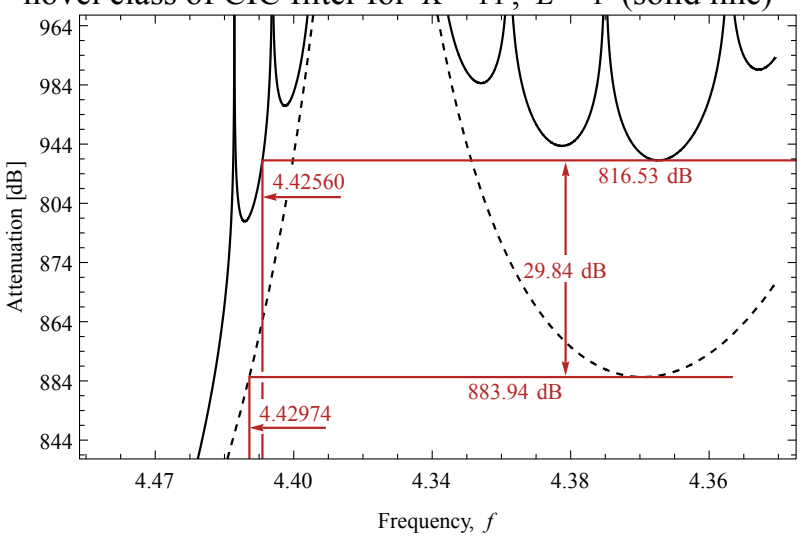

(b) Classical CIC filter for $K=18$ (dashed line), novel class of CIC filter for $K=18, L=2$ (solid line)

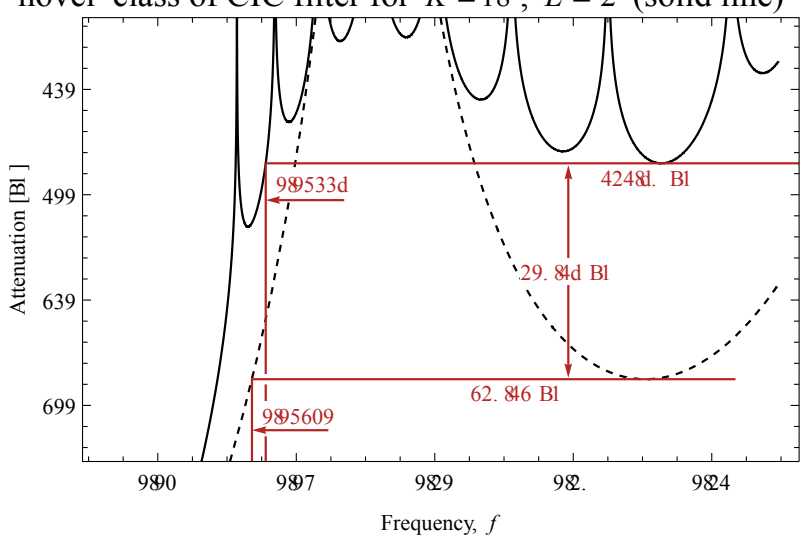

(c) Classical CIC filter for $K=25$ (dashed line), novel class of CIC filter for $K=25, L=3$ (solid line)

Fig. 3. Zooms of normalized magnitude response characteristics in $\mathrm{dB}$ of classical CIC filter (dashed lines), and proposed novel class of CIC FIR filter functions (solid lines), for $N=11$.

The filter functions have the same level of constant group delay, as well as the number of delay elements, but the novel first designed class gives higher insertion losses in stopband, and it has higher selectivity.

Zooms of the normalized magnitude response characteristics of classical CIC filter and proposed class of CIC FIR filter functions are given in Figs. 2 and 3, for different values of parameter $N$. The novel class of CIC filter functions has two peaks in the transition area of the classical filter (at a frequency between the passband $f_{c p}$ and stopband $f_{c S}$ cut-off frequencies). Note that the attenuations of the novel class in the stopband area are higher than the attenuations of the classical CIC filter in the stopband area. For $N=6$, in case of $K=11$ (Fig. $2 a$ ), a classical CIC filter has an attenuation of $136.68 \mathrm{~dB}$ and a novel class $166.52 \mathrm{~dB}$. An improvement of $29.84 \mathrm{~dB}$ or approximately about $21.8 \%$ is achieved. In case of $K=18$ (Fig. 2b), an improvement of $34.90 \mathrm{~dB}$ or approximately about $15.6 \%$ is achieved. In case of $K=25$ (Fig. 2c), a significant improvement of $39.82 \mathrm{~dB}$ or approximately about $12.8 \%$ is achieved. For $N=11$, in case of $K=11$ (Fig. $3 a$ ), the achieved improvement is $43.19 \mathrm{~dB}$ or approximately about $33.73 \%$. In case of $K=18$ (Fig. $3 b$ ), the improvement is $73.20 \mathrm{~dB}$ or about $33.08 \%$. For $K=25$ (Fig. $3 c$ ), the improvement is 102.49 $\mathrm{dB}$ or approximately about $32.80 \%$. Note that for a higher filter order significant improvements of about $33 \%$ are achieved. From Figs. 2 and 3, it is also evident that the normalized stopband cut-off frequencies are practically identical for different values of integer parameter $L$, but minimum atttenuation increases rapidly by increasing its value.

The locations of zeros in the $z$-plane along with their multiplicities for classical and proposed filter functions are shown in Figs. 4 and 5, for $K=18$ cascades and case of $N=6$ and $N=9$, respectively. The CIC filter function has $N-1$ different zeros, $z_{r}=e^{j \cdot 2 \pi \cdot r / N}, r=1,2, \ldots, N-1$ . All zeros are multiple with multiplicity $K$ and lie on the unit circle. The total number of zeros is $N \cdot K$. The zeros of the proposed filter functions are more evenly distributed with their multiplicities and therefore reduced, as can be seen in Figs. $4 b$ and $5 b$.

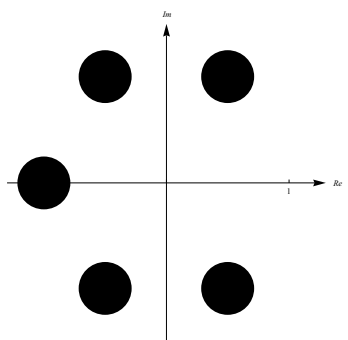

(a) Classical CIC filter

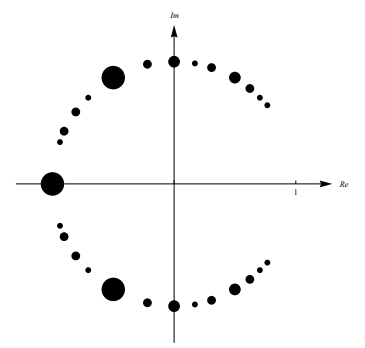

(b) Proposed filter, $L=2$

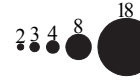

Fig. 4. Locations and multiplicities of filter function zeros in $z$-plane for $N=6$, and $K=18$ cascades

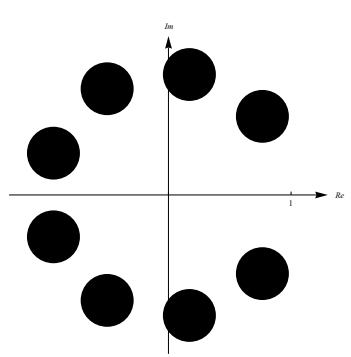

(a) Classical CIC filter

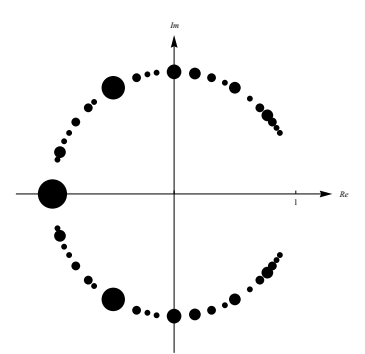

(b) Proposed filter, $L=2$

\section{$2.34580^{10}$}

Fig. 5. Locations and multiplicities of filter function zeros in $z$-plane for $N=9$, and $K=18$ cascades. 
Improvement of the attenuation in the stopband of the proposed novel class of CIC filter functions over classical CIC filters, versus filter order $N \in\{5-20\}$, obtained for different values of parameter $L$ is shown in Fig. 6. Data given in Fig. 6 show that the novel filter class has much better performances than the classical CIC filter, especially for lower filter orders $N \in\{5-13\}$.

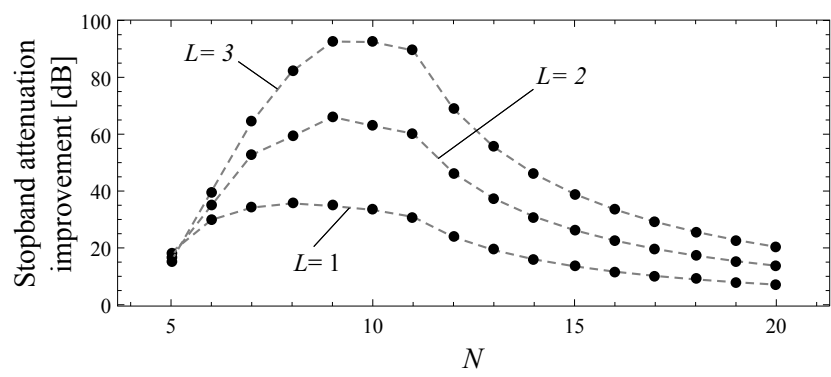

Fig. 6. Improvement of the attenuation in the stopband of the proposed novel class over classical CIC filters.

Fig. 7 presents two-dimensional (2D) contour plots of normalized magnitude response characteristics (overall and lower frequency part zoomed) of the classical CIC filters and the proposed novel class of CIC FIR filter functions. As the value of the parameter $N$ increases, as well as the normalized frequency, the benefits of the proposed filter class become less apparent, and the characteristics closely resemble those of the classical CIC filters. Therefore, it can be concluded that the proposed filter class is more efficient in the lower part of frequency range and for smaller values of parameter $N$.

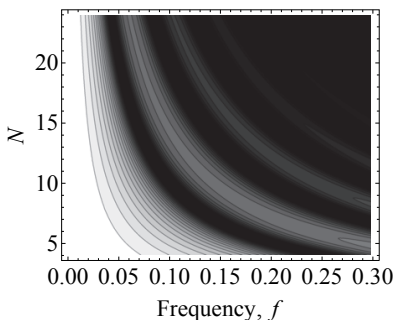

(a) Classical CIC filter with $K=18$

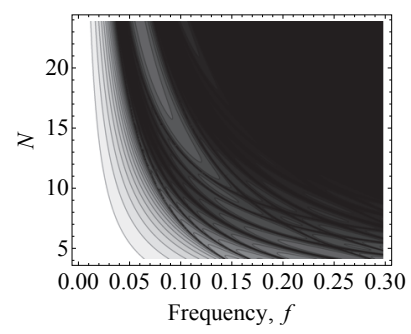

(c) Proposed filter class with $K=18, L=2$

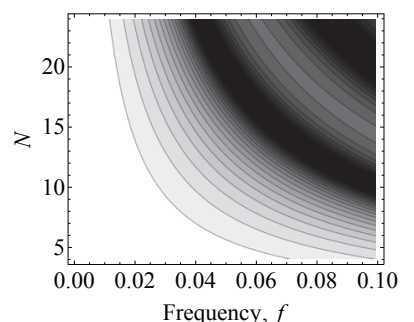

(b) Classical CIC filter zoom of lower part of frequency range

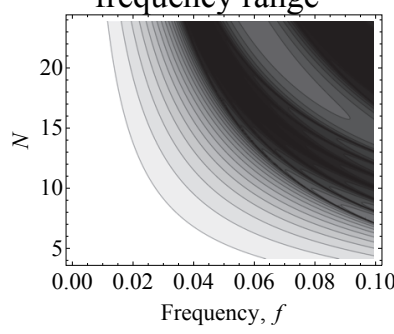

(d) Proposed filter class zoom of lower part of frequency range

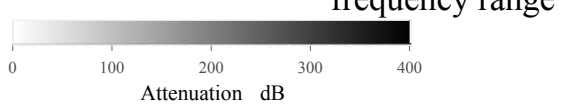

Fig. 7. 2D contour plots of magnitude frequency response characteristics for classical and proposed first class of CIC FIR filters for $N \in\{4-24\}$ and $K=18$.

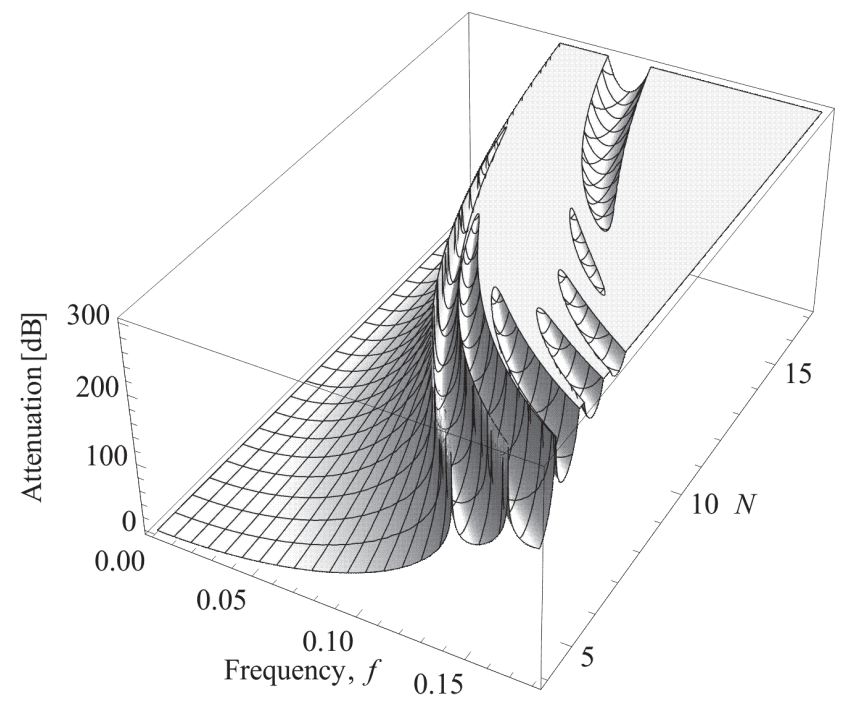

Fig. 8. 3D plot of normalized attenuation response characteristic in $\mathrm{dB}$ of the proposed first class of CIC FIR filter for $N \in\{4-17\}$, and $K=18$ obtained for $L=2$.

In Fig. 8, a three-dimensional (3D) plot of normalized magnitude response characteristic of a novel class of CIC FIR filter functions is shown. The Figure shows a normalized magnitude response in frequency domain as a function of parameter $N \in\{4-17\}$, for case of $K=18$. It is worth noting that with the increase in the value of the parameter $N$ the passband becomes narrower, as is expected. The number of transfer function zeros is increased and this is clearly visible in the branching of high loss regions in magnitude response characteristics, especially for the smaller values of the parameter $N$ and towards higher frequencies.

\section{CONCLUSION}

This paper proposes a novel class of CIC FIR filter functions which is designed based on the classical CIC filters, by spreading the delays in the CIC filter comb stages. The delays are set around one specific value of filter order $N$.

An important measure of the superiority of the proposed filter class performances is to compare the proposed filter characteristics to the characteristics of the classical CIC filters. A way to do a fair comparison is to design the filter functions for the same value of the group delay. Performance comparisons are done with a few design examples. The differences of the attenuations of the proposed modified filter class and the classical CIC filters are given to show the achieved improvement of the attenuation in $\mathrm{dB}$ in the stopband.

The possibilities of the application of filters in modern communications systems are very wide. They can be used in software defined radio systems [2] - [3], FPGA design [4], sigma-delta analog-to-digital converters [5] - [6], [19] - [22], etc.

The novel CIC filter functions have integer coefficients of the impulse responses and linear phase response characteristics. For a proper understanding of complex phenomena in pharmacy, medicine and cosmos, it is necessary to use filters with a linear phase response and a 
maximum possible attenuation in the stopband region. In some applications, the passband of the filter needs to be extremelly small. New demands of high-resolution devices will be largely defined by the quality of filter function. In the 70's, professional equipment was able to perform measurements up to $160 \mathrm{~dB}$.

\section{ACKNOWLEDGMENT}

The authors would like to thank Prof. Dr. Ljiljana Milić, Prof. Dr. Branimir Reljin, Prof. Dr. Miodrag Popović, Prof. Dr. Miroslav Lutovac of the School of Electrical Engineering, Belgrade, Serbia and Prof. Dr. Vladimir M. Stojanović of the Research Laboratory of Electronics, Massachusetts Institute of Technology, Cambridge, USA.

\section{REFERENCES}

[1] E. B. Hogenauer, "An economical class of digital filters for decimation and interpolation", IEEE Transactions on Acoustics, Speech and Signal Processing, pp. 155-162, April 1981.

[2] W. A. Abu-Al-Saud, G. L. Stuber, "Modified CIC filter for sample rate conversion in software radio systems", IEEE Signal Processing Letters, vol. 10, no. 5, pp. 152-154, May 2003.

[3] G. Jovanović Doleček, "Modified CIC filter for rational sample rate conversion", ECTI Trans. on Computer and Information Technology, vol. 4, no. 1, pp. 15-20, May 2010.

[4] K. Kalyani, S. Siva, S. Rajaram, "FPGA implementation of high speed CIC filter for software defined radio system", $A S A R$ International Conference, Coimbatore Chapter, 12 May, pp. 92-95, 2013.

[5] L. L. Presti, "Efficient modified-sinc filters for sigma-delta A/D converters", IEEE Trans. on Circuits and Systems II: Analog to Digital Signal Processing, vol. 47, no. 11, pp. 1204-1213, 2000.

[6] M. Laddomada, "Comb-based decimation filters for $\Sigma \Delta$ A/D converters: Novel schemes and comparisons", IEEE Trans. on Signal Processing, vol. 55, no. 5, pp. 1769-1779, 2007.

[7] G. Jovanović Doleček, S. K. Mitra, "A simple method for the compensation of CIC decimation filter", Electron. Letters, vol. 44, no. 19. pp. 1162-1163, 2008.

[8] G. Jovanović Doleček, "A simple wideband CIC compensator", Electron. Letters, vol. 45, no. 19, pp. 1270-1272, 2009.

[9] G. Jovanović Doleček, F. Harris, "Design of wideband CIC compensator filter for a digital IF receiver", Digital Signal Processing, vol. 19, pp. 827-837, 2009.

[10] A. Fernandez-Vazquez, G. Jovanović Doleček, "Maximally flat CIC compensation filter: Design and multiplierless implementation”, IEEE Trans. Circuits Syst. II, vol. 59, no. 2, pp. 113-117, 2012.

[11] G. Jovanović Dolecek, A. Fernandez-Vazquez, "Novel droopcompensated comb decimation filter with improved alias rejections", AEU - International Journal of Electronics and Communications, vol. 67, pp. 387-396, 2013.

[12] G. Jovanović Doleček, A. Fernandez-Vazquez, "Trigonometrical approach to design a simple wideband comb compensator", $A E U$ International Journal of Electronics and Communications, vol. 68, no. 5, pp. 437-441, May 2014.

[13] J. O. Coleman, "Chebyshev stopbands for CIC decimation filters and CIC-implemented array tapers in 1D and 2D", IEEE Trans. Circuits Syst. I, vol. 59, no. 12, pp. 2956-2968, 2012.

[14] B. P. Stošić, V. D. Pavlović, D. N. Milić, "Comparison of novel designed class of CIC FIR filter functions with classical CIC filters", XLIX International Scientific Conference on Information, Communication and Energy Systems and Technologies - ICEST 2014, Serbia, Niš, June 25- July 27, vol. 1, pp. 161-164, 2014.

[15] V. D. Pavlović, D. N. Milić, B. P. Stošić, "Characteristics of novel designed class of CIC FIR filter functions over classical CIC filters", $1^{\text {st }}$ International Conference on Electrical, Electronic and Computing Engineering - IcETRAN 2014, Serbia, Vrnjačka Banja, June 2-5, pp. EKI1.5-1-5, 2014.

[16] B. P. Stošić, V. D. Pavlović, "On design of a novel class of selective CIC FIR filter functions with improved response", $A E U-$ Intern. Journal of Electronics and Communications, vol. 68, no. 8, pp. 720-729, 2014

[17] B. P. Stošić, V. D. Pavlović, "Design of selective CIC filter functions", AEUE - Intern. Journal of Electronics and Communications, http://dx.doi.org/10.1016/j.aeue.2014.07.002

[18] D. N. Milić, V. D. Pavlović, "A New Class of Low Complexity Low-Pass Multiplierless Linear-Phase Special CIC FIR Filters", IEEE Signal Processing Letters, vol. 21, no. 12, pp. 1511-1515, 2014.

[19] G. Molina Salgado, G. Jovanović Doleček, J. M. de La Rosa, “An overview of decimator structures for efficient sigma-delta converters: Trends, design issues and practical solutions", 2014 IEEE Intern. Symposium on Circuits and Systems (ISCAS), 1-5 June 2014, pp. 1592-1595.

[20] A. Kilic, D. Haghighitalab, H. Mehrez, H. Aboushady, "Low-power comb decimation filter for RF Sigma-Delta ADCs", 2014 IEEE Intern. Symposium on Circuits and Systems (ISCAS), 1-5 June 2014, pp. 1596-1599.

[21] F. J. Harris, "Reduce energy requirements by coupling a poly-phase pre-filter and cic filter in high-performance Sigma-Delta A/D converters", 2014 IEEE Intern. Symposium on Circuits and Systems (ISCAS), 1-5 June 2014, pp. 1600-1603.

[22] J. O. Coleman, "Integer-coefficient FIR filter sharpening for equiripple stopbands and maximally flat passbands", 2014 IEEE Intern. Symposium on Circuits and Systems (ISCAS), 1-5 June 2014, pp. 1604-1607. 